\title{
Mobile Technology as an ICT Solution for Crisis Management: An ABET IS Knowledge Interest Area Service Learning Case Study
}

\author{
Frank Hartle, Robert Morris University, fxhst270@mail.rmu.edu \\ ChristopherWydra, Robert Morris University, cawst157@mail.rmu.edu \\ Frederick Kohun, Robert Morris University, kohun@rmu.edu \\ Daniel Rota, Robert Morris University, rota@rmu.edu
}

\begin{abstract}
This paper highlights the continued issues of interoperability communication during a multiagency response to a crisis incident as a fundable applied curriculum based case study. With the proliferation of mobile ICT devices such as smart phones, tablets, netbooks and laptops into society and their diffusion into our professional and personal lives, one has to ask why we still use two-way wireless radio developed in the 1890's to communicate during crisis events. Leveraging off-the-shelf mobile ICT, this paper, in support of the current ABET IS curriculum,proposes further developing Internet Protocol systems to replace the current disparate radio based and multilayered proprietary communication systems that currently exist. The ABET IS curricular subjects of usability/technology acceptance, human factors, software/applications, hardware/mobile technology, networks/internet considerations, and security become part of the learning and discussion to contend with real time issues in need of a solution.
\end{abstract}

Keywords: ICT, IP, Interoperability, Long Term Evolution, Mesh Network and ABET curriculum

\section{INTRODUCTION}

The communication problems that plagued the response to the September 11, 2001 attacks (Kean et al, 2005), the confusion and lack of communication after hurricane Katrina (Miller, 2006) and the recent uptick in violent shooting events with multiple victims have highlighted the need, once again, to fix our emergency responder communication network. The 9/11 Commission made recommendations to change how local, state and federal emergency responders communicate due to the disastrous communication failures during the 9/11 attacks (Kean et al, 2005). Unfortunately, little has been done to fix the issue in the last twelve years. While much effort has been undertaken by the federal government, multiple and competing proprietary systems are being developed that require substantial financial investment at the local and state level (SAFECOM, 2013). To date, local state and federal emergency responders are still utilizing separate radio frequencies and incompatible communication equipment (Lee, 2013). This is due to a lack of a national standard and separate purchasing driven by cost and funding across multiple jurisdictions (Weiser, 2006). This problem serves as a service learning like case study as part of an ABET curricular IS knowledge interest area. The ABET IS curricular subjects of usability/technology acceptance, human factors, software/applications, hardware/mobile technology, networks/internet considerations, and security become part of the learning and discussion to contend with real time issues in need of a solution.

\section{History of Radio (Two - Way Communication)}

The mobile two-way radio enables people to converse using a radio frequency. The radio can transmit and receive messages while operating on the same radio frequency. The use of two-way radios began just before World War II. Two-way radios, which, by the early '60s, had improved so that the channel bandwidth required transmitting a voice signal reduced from $120.0 \mathrm{kHz}$ to $30 \mathrm{kHz}$. These early systems allowed only one conversation per channel, and users had to scan channels manually to find a free channel (Nijhawan, 2000). Figure 1 illustrates the operation of the twoway radio. 


\section{Issues in Information Systems}

Volume 14, Issue 2, pp.429-437, 2013

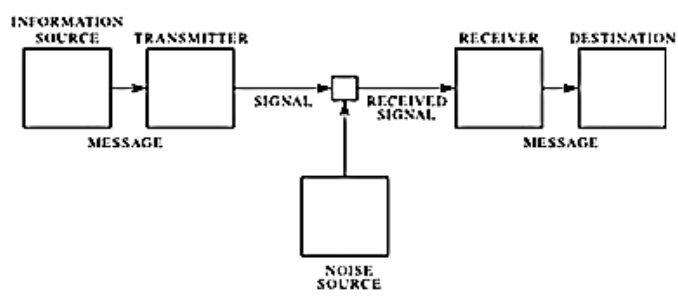

Figure 1 The operation of the two-way radio

The reliability between two-way radios relies on the quality and conditions of the radio channel frequency. Radio frequency (RF) channels facilitate the wireless data communication system of the two-way radio. Channel conditions, the quality of the transmission, can vary due to external forces, such as; building, obstacles, power variations, and environmental conditions (Needham, 1998).

\section{History of Shannon Weaver}

Two-way radio transmitting and receiving using a RF is predicated on the basic communication model. The communication model that is well recognized is depicted by the Shannon Weaver "transmission" model. The best known model of the communication process is the one postulated by Claude Shannon and Warren Weaver in 1949. The model was originally developed to explain electronic transmission of data; the Shannon Weaver model has been well used because of its simplicity and its foundation in scientific principle (Bowman, 1987, p. 23).

Figure 2 illustrates the Shannon Weaver transmission model:

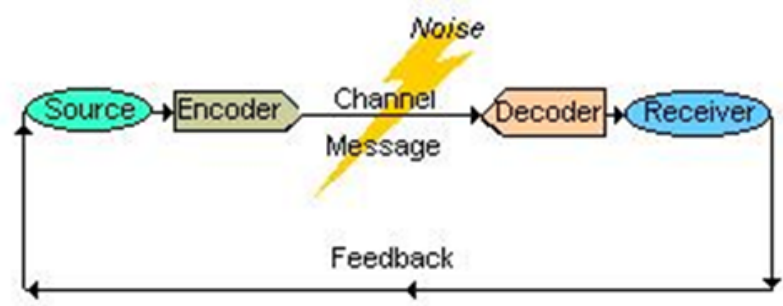

Shannon-Weaver Model

Figure 2

Since the inception of the Shannon Weaver model, there have been many analysis of the model, which has conveyed strengths and weaknesses. The model exudes a low level communication process; a base model for the transfer of information. Because human communication and information transfer can be complicated, there are sender and receiver barriers in the communication process that affect the transmission model. Barriers from the sender's point of view are ambiguity, polarization, generalization, egocentrism, loquacity, secret mania, and distortions. The receiver also experiences barriers such as: egocentrism, message overcharge, worries, and quick thinking (Dobra, 2008, p. 18-19). Sender and receiver barriers are both contributors to the loss of information during the communication transmission.

\section{Various and Incompatible Ways Emergency Responders Communicate}

Two-way radio transmission and the Shannon Weaver communication transfer model are processes used by emergency responders during crisis. There are several examples to illustrate the various ways emergency responders communicate and how incompatible the means of communications are during emergency situations. Some examples that inhibit communications during an emergency responder crisis are non-compatible and inoperable communication systems when multi-jurisdictional organizations are involved; power and connectivity complications, and geographical issues. 


\section{Issues in Information Systems \\ Volume 14, Issue 2, pp.429-437, 2013}

If communication fails, a potential solution for quickly restoring a communication infrastructure is a hybrid wireless mesh network. Even though the communication infrastructure is established, there are issues that contribute to the lack of information transfer during the communication process. Only a comprehensive approach involving solutions for technological, sociological, and organizational issues can provide a reliable communication system during crisis situations (Manoj, 2007, p. 52-53).

The terrorist attacks in 2001 and Hurricane Katrina in 2005 are notable examples of emergency crisis situations that involved multi-jurisdiction emergency responders. The two examples greatly demonstrated communication breakdowns and incompatible ways of communication during emergency response situations. These situations demonstrated that continuity of communications, capacity, and inoperability are the primary areas of vulnerability in emergency communications that persist in communities across the country (Catellier, 2008, p. 485).

\section{New Technologies Developed for Emergency Responders}

Federal, State, and Local agencies are well aware of communications inoperability during emergency responses to crisis situations. New technologies and systems are being developed for emergency responders. Communication and the ability to coordinate actions among emergency team members in sensitive environments involving multiple jurisdictions is the key to an effective response. Mobile communication networks integrated within a human-system interaction environment can handle the collaborative aspects of responding to a crisis; this would provide a crisis response system with the requisite intelligence to support crisis response workers in an emergency (Yuan, 2005, p. 95).

Digital communication using IP-enabled networks is another technological initiative that would best be supported by Next Generation 911 technology (NG 9-1-1) to upgrade 911 emergency call centers. The IP-enabled networks rely on the same type of home and business Internet network connections and can share capacity with other using a broadband connection. Deploying NG 9-1-1 is not considered cost effective (Moore, 2001, p. 181).

\section{INTERNET PROTOCOL AND INFORMATION COMMUNICATION TECHNOLOGY}

Although the Web is sometimes one and the same with the Internet in people's minds, the two words are distinct, and refer to different but interrelated concepts in the computerized telecommunication system. The Internet is an interconnected pool of small computer networks that have been wired together and allow electronic information to be sent from one network to the next around the world (Zhong Zhou, 2000).

The Web,best known for its ability to combine text with graphics and other multimedia on the Internet, is one type of high-level data exchange protocols on the Internet.Prior to the inception of the Web, there was Telnet, the traditional command-driven style of interaction, FTP, a file transfer protocol useful for retrieving information from large file archives, Usenet, a communal bulletin board and news system, andthere was also e-mail for individual information exchange, and e-mail lists, for one-to-many broadcasts. In addition to the above mentioned mediums of data transfer and retrieval, Gopher was used to provide campus-wide information system sharing among universities and research institutions, and WAIS, a powerful search and retrieval system developed by Thinking Machines, Inc. In 1990 Tim Berners-Lee and Robert Cailliau at CERN (www. cern.ch), the European Laboratory for Particle Physics, created a new information system called "World Wide Web" (WWW)(Zhong Zhou, 2000).

In addition, the Web has some other key features that make it stand out from earlier Internet information exchange protocols. Since the Web came later to the Internet than previous data transfer protocols, it has to be compatible backwards with other communications protocols in addition to its native language, HyperText Transfer Protocol (HTTP). Among the foreign languages spoken by Web browsers are Telnet, FTP, and other high-level communication protocols mentioned earlier. The support for foreign protocols lets users use a piece of software, the Web browser, to access information without shifting from protocol to protocol and software incompatibility(Zhong Zhou, 2000).

\section{Proliferation of Mobile ICT Used At Home}




\section{Issues in Information Systems \\ Volume 14, Issue 2, pp.429-437, 2013}

Increased access to and use of Information and Communications Technology (ICT) will increase users spatial extensibility (or reach), even stimulating travel and fuelling the time space convergence of society, thus transforming the patterns of localization in a sprawling and globalizing direction (Janelle \& Gillespie 2004). An often overlooked fact, however, is that ICT use is also a stationary, mainly place-bound and home centered activity. ICT competes with other in-home and out of- home activities, not least the consumption of media - TV, video, DVDs, CDs, etc. - and social interaction with family and nearby friends in real life (Thulin 2004; Thulin\&Vilhelmson 2006).

ICT and virtual mobility have made the walls of the home permeable, being stationary and face-to-face contact as principles of the organization, location, and co-ordination of human activity and impacting the role of the home and other anchoring points of everyday life (Vilhelmson, \&Thulin, 2008).

The competition between mobility's for users use of time and space is an important context for the emerging use of ICT (Hanson 1998; Gershuny 2002; Ellegård\&Vilhelmson 2004; Thulin\&Vilhelmson 2006). From the perspective of an individual's daily activity pattern, it may be illustrated by an extended time-geographical model. Figure 3 depicts the trajectory of a user's by the end of 2007thatused various space-transcending technologies during the course of a day.

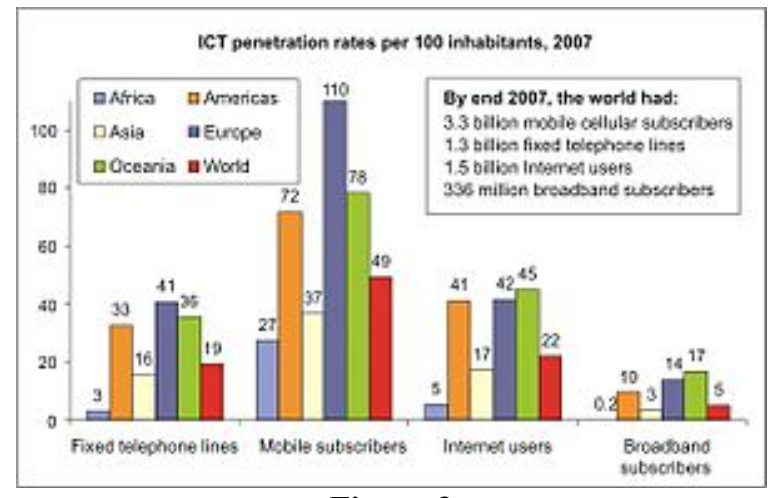

Figure 3

\section{Proliferation of Mobile ICT Used In Business}

Today's - always on, always best connected, seamless connectivity - mantra is more to do with this rich legacy as a network provider than any other driver (Dennis, 2007).

Any use of mobile communication technology for virtual business organizing must take into account the central role of communication: "Communication is fundamental to any form of organizing, but it is preeminent in virtual organizations" (DeSanctis,\&Monge, 1998). Physical space and human behavior become subordinate elements in the support and process of communication, which is constant as users move through different environments and perform various behaviors. As users of mobile ICTs, people can become part of social networks that develop group meanings and usage patterns by communicating with one another through information technologies (Gallant, 2005).

Figure 4 represents a staircase that builds from the user's desire for mobility at the bottom to a trust/risk-based approach to maintaining the ongoing relationship at the top. It has the potential to underpin more radical business models (Dennis, 2007). 


\section{Issues in Information Systems \\ Volume 14, Issue 2, pp.429-437, 2013}

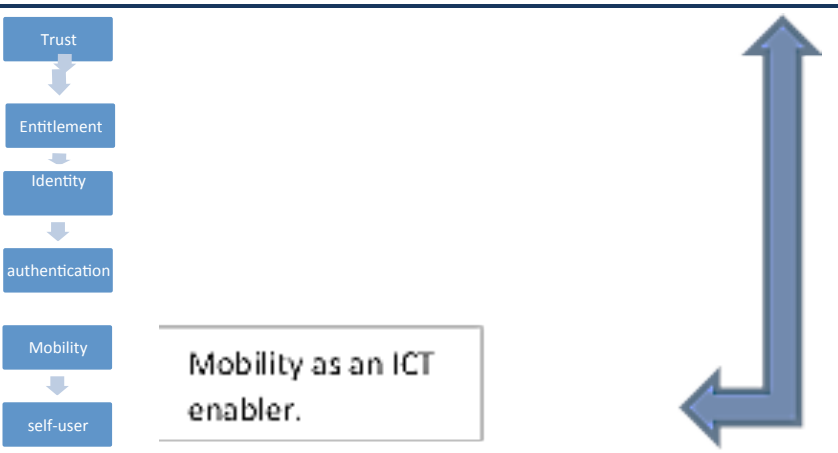

Figure 4

Particular challenges faced in ICT business include product complexity being increased due the products containing a combination of mechanics, electronics, and software. This is added with rapid technological developments and requirements to generate new products rapidly (Saaksvuori\&Immonen, 2008).

\section{MOBILE ICT AND EMERGENCY COMMUNICATION CONVERGENCE}

With the proliferation of mobile ICT both at home and in business and with internet usage continuing to grow, now nearing 85\% among Americans, (Pew, 2013) serious consideration to develop IP based communication systems and networks has begun. In preparation for the development and utilization ofa nationwide wireless system for commercial and public use,the United States Congress passed the Digital Television and Public Safety Act of 2005 to free up bandwidth (FCC.gov, n.d). The Federal Communications Commission (FCC) adopted the rules to establish a $700 \mathrm{MHz}$ public safety band to establish a nationwide broadband network for public safety use upon the vacant frequencies in 2009(FCC.gov, n.d). In October of 2009, the United States Congress requested the Implementation Coordination Office to develop a plan to migrate from the analog 911 system currently in use to a new IP based Next Generation 911 system now call NG9-1-1 (NTIA, 2009). In, 2010 the FCC recommended a public -private partnership that would allow commercial use of the new frequencies. In return, the government would be allowed to utilize the commercial network infrastructure for public safety use (FCC, 2010). The next year the White House announced President Obama's support for a wider swath of frequency and backing for additional funding for a national wireless public safety network saying:

"In his State of the Union address, President Obama set the goal of enabling businesses to provide high-speed wireless services to at least 98 percent of all Americans within five years. The rollout of the next generation of highspeed wireless - the " $4 G$ " technology now being deployed in the United States by leading carriers-promises considerable benefits to our economy and society. More than 10 times faster than current high speed wireless services, this technology promises to benefit all Americans, bolster public safety, and spur innovation in wireless services, equipment, and applications. "(Whitehouse.gov, 2011)

In November of 2011, the Department of Homeland Security outlined a conceptual framework (Figure 5) of how the transition from the current technologies would evolve and converge. The IP enabled system would use Long Term Evolution or LTE technology to transfer data on the new national network. The decision to use LTE was based on consensus from public safety officials, the government and commercial network experts and the fact that the commercial wireless carriers have largely moved to IP enabled LTE networks (Moore, 2012). Figure 5 shows this LTE evolution.

The parallel development of the NG9-1-1 center and the national public safety broadband network to connect public safety to the NG9-1-1 would allow for full IP integration of the current analog system. 


\section{Issues in Information Systems \\ Volume 14, Issue 2, pp.429-437, 2013}

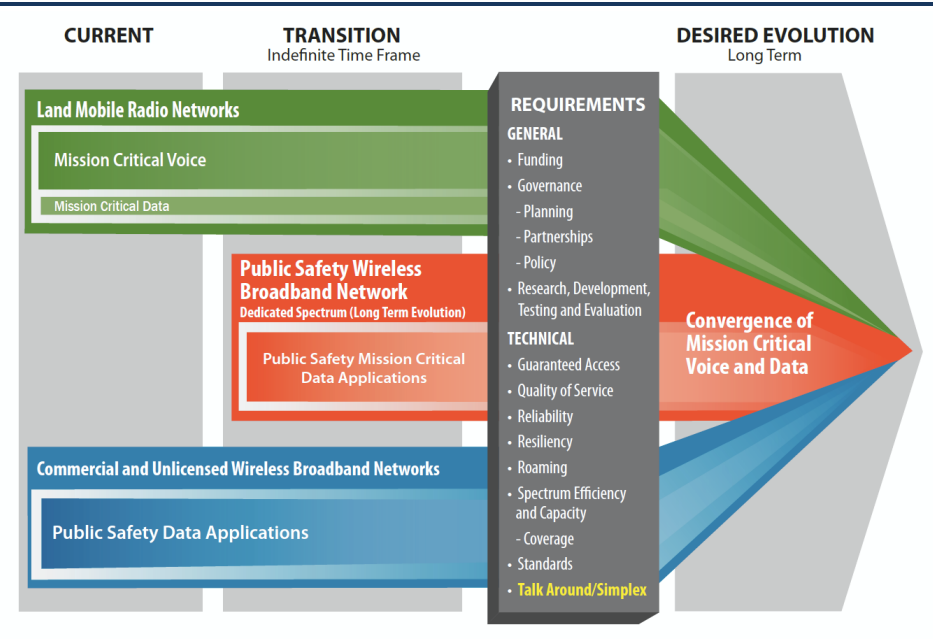

Figure 5 (Public Safety Communication Evolution. (2011). Retrieved January 21, 2013, from: URL (http://www.illinois.gov/firstnet/PublicSafety/Pages/default.aspx))

The benefits of such a system are many. For instance, although the mobile ICT hardware may change, the data transfer platform (IP enabled LTE) would remain the same. This is important since hardware (phones, tablets and computers) change rapidly and can be exchanged without incorporating a new information delivery system. The proliferation of mobile ICT devices makes it easily learned by the users and due to the great supply of makes and models, the cost is considerably lower. For example it costs is $\$ 4,400$ per officer to carry a Motorola XTS 5000 portable radio compared to about $\$ 500$ for a high end smart phone that does considerably more(Moore, 2012).

\section{APPLICATION TO ABET IS CURRICULAR AREAS OF STUDY}

\section{Hardware}

As we move closer to the realization of a fully integrated IP based communication systems for public safety, the development of future competencies will be needed to support research and development of the multiple software and hardware configurationsfor the mobile ICT devices.

These mobile ICT devices may includesmart phones, wearable mobile ICT, tablets and laptops. In order to use such devices, mobile apps will be need to be developed. Maximizing the opportunities of a broadband network allows for the creation of mobile ICT applications that can potentially link each responder to the command center (Moore, 2011). DHS (2011), compiled apartial list of critical applications that public safetypersonnel wanteddeveloped to work on the new network and incorporated into any new ICT that would be used. These include,automatic vehicle location, incident command white board, aerial video, vehicle-mounted video, helmet cameras, third-party camera resources, mobile data computers, patient, evacuee, and deceased tracking, internet connection, bio telemetry, and third-party sensors (p.5). The testing of device reliability and security are another area that must be studied.

\section{Software}

In conjunction with ICT applications, web APIs that allow the app content to be transferred and shared on a secure website, or in this case a command dashboard, would also need to be considered. The development of these two components would require additional skills. For example, Mobile ICT applications (app) would have to be developed by downloading the software development kits (SDK) for the four main providers of mobile ICT. This would include Android, Apple, Microsoft and Blackberry. The future apps would need to be developed to interface with a dedicated website or in our case the web API. Potential research includes the development of anapp that could be programmed to act as sort of an emergency response Facebook but with the ability to stream live video feeds, to push to talk, text, and email or create side conversations via teleconference. Each member could be represented on the screen as an icon. Many civilian apps already enable such capabilities. The benefit to this would 


\section{Issues in Information Systems \\ Volume 14, Issue 2, pp.429-437, 2013}

be the familiarization and wide spread use of social apps and the ability to see and talk with multiple responders at the same time. Special add-ons may be developed in order to allow responders to use wearable devices or operate their devices hands free. For example, a smart watch could be used to start and stop transmission of a voice signal or to stop or start streaming video. The app could be programed to interact with the wrist device by tapping it or by the responder shaking his/her wrist a certain way to activate the communication process. In addition, the emergency commanders could activate any device to locate missing responders or to see what they see.

The web API dashboard would be programmed to connect multiple parties who link into the site via the mobile ICT apps. Each supervisor would have access to their own responders and the main command post would have access to all responders. The dashboard would be hosted on the local machine and also backed up on a local server to enable the collection and memorization of the incident data such as conversations and videos. Much like other social web APIs it could host hundreds of user simultaneously.

\section{Mesh Networks}

In addition to researching and developing software applications and dashboards, wireless mesh network could be developed based on a theory proposed by Portmann\&Pirzada (2008). They proposed that every emergency vehicle should be equipped with a mesh router that is powered by the vehicle battery. Ideas about redundant and scalable mesh networks that work regardless of the power infrastructure are another area of potential research. This network could interact with the dashboard creating a closed mobile Incident Area Network (IAN). Each mobile ICT device could contain the above-mentioned app. The app (when opened) would connect the responding officers to the dashboard and from the dashboard to all the other responders. This would allow each commander to see and talk to each IAN member. The emergency responders could talk, post video or photos, email or text to all incident participants or to an individual depending on the need and set permissions. Voice, text and video could be wirelessly shared with the incident command vehicle and pushed out to other agencies via an internet gateway if needed (Portmann\&Pirzada, 2008) or the IP LTE network.

Another plus to the system is that alerts can be sent directly to the public via social media. Civilian versions of the apps could be developed to allow emergency responders to communicate directly with the public and vice versa. . [Potential area for student development]

\section{Technology Acceptance}

There are several areas that need to be researched as it pertains to technology acceptance. These include political, cultural and technical matters. Culturally, emergency responders have been accustomed to communicating by twoway radio for much of the last 70 years. Research into the reliance on two-way communication and its acceptance, or not, of the new IP based system that potentially could allow simultaneous voice and video would be beneficial prior to heavy investment into the network.

\section{CONCLUSIONS}

This case study of mobile technology as an ICT solution/tool for crisis management serves two purposes in the context of an ABET-CAC IS curriculum. First all, it provides a focal point for integrating multiple IS curricular subject areas to facilitate/support a contemporary public security problem-crisis management. Crisis management and public safety/security is front page news - particularly in light of the Boston Marathon Terror Attack. This case provides a service learning focus to incorporate the issues associated with crisis management communication through technology. The ABET IS curricular subjects of usability/technology acceptance, human factors, software/applications, hardware/mobile technology, networks/internet considerations, and security become part of the learning and discussion to contend with real time issues in need of a solution. Secondly, it brings real time relevance to the IS curriculum with value added beyond a degree and job. It focuses on cooperative problem solving of a problem which is recent and without precedent. The communication issues in crisis management inherently force systems thinking that involves both technical and behavioral subject inter-disciplinarity. Finally, because of the urgency and universal nature of this emerging problem, there is immense funding potential to re-develop curricula to teach, learn, experience, serve, as well as to research solutions that go beyond the classroom and provide value added to society in general. 


\section{Issues in Information Systems}

Volume 14, Issue 2, pp.429-437, 2013

\section{REFERENCES}

A National Plan for Migrating to IP-Enabled 9-1-1 Systems | NTIA. (2009.).NTIA. Retrieved May 10, 2013, from http://www.ntia.doc.gov/report/2009/national-plan-migrating-ip-enabled-9-1-1-systems

Bowman, J. P., \&Targowski, A. S. (1987). Modeling the Communication Process: The Map is Not the Territory. Journal of Business Communication, 24(4), 21-34.

Catellier, A. \&Voran, S. (2008). Emergency Communications: Vulnerabilities Remain and Limited Collaboration and Monitoring Hamper Federal Efforts. (2011). Journal of Communications Research, 3(4), 481-545.

Current Projects. (2013). SAFECOM. Retrieved February 2, 2013, from http://www.safecomprogram.gov/currentpr

Dennis, R. (2007). Mobility as an ICT Enabler. BT TechnologyJournalVol 25 No 2 April 2007.

Department of Homeland Security. Interoperability Planning for Wireless Broadband. Informational Guide,

Washington: Department of Homeland Security,(2011), from

http://www.safecomprogram.gov/library/lists/library/DispForm.aspx?ID=331

DeSanctis G, Monge P (1998) Communication processes for virtual organizations. J Comput Mediated Commun 3(4), from the World Wide Web http://www.ascusc.org/jcmc/vol3/issue4/desanctis.html\#Communication

Dobra, A., \&Popescu, A. (2008). Barriers in Verbal Communication. Scientific Bulletin of the Politehnica University of Timisoara. Transactions On Modern Languages / BuletinulStiintific Al UniversitatiiPolitehnica Din Timisoara. SeriaLimbiModerne, 7(1/2), 15-20.

Federal Communications Commission (n.d) 700 Mhz Public Safety Spectrum. Public Safety and Homeland Security Bureau. Retrieved May 2, 2013, from transition.fcc.gov/pshs/public-safety-spectrum/700-MHz/

Federal Communications Commission. (2010). Connecting America: The National Broadband Plan. Washington, $D C$.

Gallant, L. (2006). An Ethnography of Communication Approach to Mobile Product Testing. Personal \& Ubiquitous Computing, 10(5), 325. doi:10.1007/s00779-005-0053-4

Hanson, S. (1998), Off the Road? Reflections on Transportation Geography in the Information Age. Journal of Transportation Geography 6, pp. 241-249.

Internet Adoption. (2013). Pew Research Center's Internet \& American Life Project. Retrieved April 1, 2013, from http://www.pewinternet.org/Trend-Data-(Adults)/Internet-Adoption.aspx

Janelle, D.G. \& A. Gillespie (2004), Space-time Constructs for Linking Information and Communication Technologies with Issues inSustainable Transportation. Transport Reviews 24, pp. 665-667

Jian-Zhong, Z. (2000). The Internet, the World Wide Web, Library Web Browsers, and Library Web Servers. Information Technology \& Libraries, 19(1), 50.

Lee, S. (2013). Allegheny County, municipal agencies get new FCC deadline for radio upgrades, TribLIVE. Tribune-Review. Retrieved February 5, 2013, from http://triblive.com/neighborhoods/yourmckeesport/yourmckeesportmore/3486887-74/county-uhfpolice\#axzz2LqmPHu43 


\section{Issues in Information Systems}

Volume 14, Issue 2, pp.429-437, 2013

Manoj, B. S., \& Baker, A. (2007). Communication Challenges In Emergency Response. Communications of the $A C M, 50(3), 51-53$.

Miller, R. (2006). Hurricane Katrina: Communications \& Infrastructure Impacts. Threats at Our Threshold: Homeland Defense and Homeland Security in the New Century, 191-204.

Moore, L. K. (2012) Funding Emergency Communications: Technology and Policy Considerations. January 5, 2012. Congressional Research Service.

Nijhawan, V. (2000). Look Ma, No Wires!Siliconindia, 4(7), 32.

Portmann, M., \&Pirzada, A. (2008). Wireless mesh networks for public safety and crisis management applications. IEEE Internet Computing, 12(1), 18-25. doi:10.1109/MIC.2008.25

President Obama Details Plan to Win the Future through Expanded Wireless Access | The White House. (2011, February 10).The White House. Retrieved May 15, 2013, from http://www.whitehouse.gov/the-pressoffice/2011/02/10/president-obama-details-plan-win-future-through-expanded-wireless-access Saaksvuori, A \&Immonen, A, "Product Lifecycle Management," Springer, Berlin, 2008. doi:10.1007/978-3-540$78172-1$

Thulin, E. (2004), UngdomarsVirtuellaRörlighet. AnvändningenavDator, Internet ochMobilitelefoniettGeografisktPerspektivThe Virtual Mobility of Young People: The Use of Computers, the Internet and Mobile Phones from a Geographical Perspective. (PhD thesis, University of Gothenburg).

Thulin, E. \& B. Vilhelmson (2006), Virtual Mobility and Processes of Displacement: Young People's Changing use of ICT, Time, and Place. NETCOM-Networks and Communication Studies 20, pp. 27-39.

T.H. Kean et al., "The 9-11 Commission Report," www.911 commission.gov/report/911Report.pdf.Hall, K. G. (2005, August 29).

Vilhelmson, B., \&Thulin, E. (2008). Virtual Mobility, Time Use and the Place of the Home. (Journal of Economic \& Social Geography), 99(5), 602-618. doi:10.1111/j.1467-9663.2008.00494.x:

http://www.cyberhub.com/research/IP_threat/Index/17

Weiser, P. J. (2006). Communicating During Emergencies: Toward Interoperability and Effective Information Management. Fed. Comm. LJ, 59, 547.

Yuan, Y. \&Detlor, B. (2005). Intelligent Mobile Crisis Response Systems. (2005). Communications of the ACM, 48(2), 95-98. 